\title{
LASSO model selection with post-processing for a genome-wide association study data set
}

\author{
Allan J Motyer ${ }^{1 *}$, Chris McKendry ${ }^{1}$, Sally Galbraith ${ }^{1,2}$, Susan R Wilson ${ }^{1,2}$ \\ From Genetic Analysis Workshop 17 \\ Boston, MA, USA. 13-16 October 2010
}

\begin{abstract}
Model selection procedures for simultaneous analysis of all single-nucleotide polymorphisms in genome-wide association studies are most suitable for making full use of the data for a complex disease study. In this paper we consider a penalized regression using the LASSO procedure and show that post-processing of the penalizedregression results with subsequent stepwise selection may lead to improved identification of causal singlenucleotide polymorphisms.
\end{abstract}

\section{Background}

For a complex disease with many causal genetic factors an analysis that simultaneously takes into account the effect of all single-nucleotide polymorphisms (SNPs) is preferable to one in which each SNP is considered separately. In particular, a simultaneous analysis of all SNPs will be able to identify those SNPs that have a strong joint effect in the presence of other causative SNPs but are not necessarily identifiable in a single-marker analysis because the effect of the SNP acting alone is not strong enough to be detected. The simultaneous analysis of all SNPs in genome-wide association studies (GWAS) for a complex disease is under active consideration; see, for example, [1-4].

We approach the problem of model selection (i.e., identification of the SNPs that are associated with the phenotype) by making use of penalized-likelihood methods [5], in particular, the LASSO (least absolute shrinkage and selection operator) procedure [6], which is briefly described in the Genetic Analysis Workshop 17 (GAW17) background paper on machine learning methods [7]. We consider a two-step model selection procedure for simultaneous analysis of all SNPs in GWAS. The first step is to apply the LASSO method for penalizedlikelihood variable selection to identify a set of SNPs for further consideration. Setting the LASSO tuning

\footnotetext{
*Correspondence: a.motyer@unsw.edu.au

'Prince of Wales Clinical School, University of New South Wales, New South Wales 2052, Australia

Full list of author information is available at the end of the article
}

parameter is somewhat arbitrary, so we set these parameters to include a relatively large number of SNPs and used a second step to refine the model by using a traditional variable selection method with the candidate SNPs selected in the first step.

We also assessed the performance of the LASSO with resample model averaging. This assessment is able to show whether SNPs in our model are the most often selected SNPs when using bootstrap samples, which suggests model stability; that is, the model is not likely to change with small changes in the data.

\section{Methods}

Our analysis was carried out for the unrelated individuals in the GAW17 data set. We used phenotype replicate 1 for model selection and considered the quantitative trait $\mathrm{Q} 1$ as the response variable. Our approach is suited only to common variants. We filtered out SNPs with a minor allele frequency (MAF) less than 0.01. We identified SNPs for inclusion in a multivariate linear model with additive SNP effects and identified non-SNP covariates with the two-stage procedure already described. In the first stage we carried out the LASSO procedure using the glmnet package [8] in the R software (http://www.R-project.org). The tuning parameter $\lambda$ was determined using a 10 -fold cross-validation (CV). The non-SNP covariates (Sex, Age, and Smoke) were included as unpenalized terms. All covariates were standardized before running the LASSO procedure. 
The second stage involves performing a stepwise selection with the SNPs selected in the first stage and was performed with both the Akaike information criterion (AIC) and the Bayesian information criterion (BIC). This selection was carried out using the step function in $\mathrm{R}$ with the full model, including all SNPs from the first stage and the three non-SNP covariates used as the initial model in the stepwise search and covariates allowed to be both deleted from and subsequently added back into the model (i.e., the direction argument of the step function was set to "both").

We also performed LASSO bagging (bootstrap aggregation, or model resample averaging) [9]. This involved taking bootstrap samples of the data (sampling with replacement with sample size equal to that of the original data) and performing the first LASSO stage. We took 100 resamples and used the same $\lambda$ parameter as for the original data for each resample. We were then able to calculate the resample model inclusion proportion (RMIP) for each SNP. The RMIP is a measure of how likely a given SNP is to be selected by the LASSO procedure if the data are perturbed.

The analysis was initially carried out without knowledge of the simulating model. We subsequently made comparison with this model.

\section{Results}

The GAW17 data set contains 24,487 SNPs from 697 individuals. Filtering out SNPs with MAF $<0.01$ leaves 6,356 SNPs. Retaining only one SNP from groups of identical SNPs left 6,321 SNPs to consider.

The phenotype simulating model included 39 SNPs in nine chromosomes, of which 7 SNPs from four chromosomes are common variants (MAF $>0.01$ ). These SNPs are presented in Table 1.

It is useful to check whether any of the SNPs in the simulating model are in high linkage disequilibrium (LD) with any other SNPs, because this may lead to SNPs showing up in the model selection in the place of the simulating SNPs. The simulating SNPs C1S6533, C14S1734, C4S1878, and C4S1884 are not in LD with any other SNP (pairwise $r^{2} \geq 0.1$ ). The SNP in highest LD with C13S431 has a pairwise $r^{2}=0.315$, but it is on a different chromosome. The SNPs C13S522 and C13S523 have their highest LD with each other (pairwise $r^{2}=0.142$ ).

Our two-stage procedure was run with two different values of the LASSO tuning parameter $\lambda$, each obtained by using a 10 -fold CV. The first parameter, termed $\lambda_{\text {min }}$, minimizes the CV error. The second parameter, termed $\lambda_{1 \mathrm{se}}$, is a stronger penalty to guard against model overfit and is the maximum value with its CV error within one standard error of the minimum $\mathrm{CV}$ error. Because the penalty parameter obtained by $\mathrm{CV}$ can vary depending on the random partitioning of observations, $\mathrm{CV}$ was repeated 10 times on replicate 1 , and the median penalty parameter was used. This resulted in $\lambda_{\min }=0.0914$ and $\lambda_{1 \mathrm{se}}=0.160$. (An alternative approach is to select $\lambda$ so that a predetermined number of SNPs are selected; however, we approached the analysis without knowledge of how many SNPs were in the simulating model.)

As already mentioned, the second step was performed with the AIC and the BIC. The model selection procedure was performed on all 200 phenotype replicates. Table 2 summarizes the average number of times that the simulating model SNPs were selected.

In Table 2 we see that if the LASSO procedure with $\lambda_{\text {min }}$ is used, on average 28.1 SNPs are selected, of which 4.1 are in the simulating model. If stepwise selection with the BIC is also used, then on average 14.3 SNPs are selected, of which 3.3 are in the simulating model. So by applying the second step, we lose on average 0.8 SNP in the simulating model, but the proportion of selected SNPs in the simulating model is improved (from $14.6 \%$ to $23.2 \%$ ). If stepwise selection with the AIC is used, then on average 21.2 SNPs are selected, of which 3.8 are in the simulating model.

We also see in Table 2 that if a LASSO procedure with $\lambda_{1 \mathrm{se}}$ is used, then on average 3.3 SNPs are selected, of which 2.2 are in the simulating model. Subsequently applying stepwise selection with either the AIC or the BIC leads to little refinement of the model. This is because in this case we have used a stricter penalty in the LASSO step, so there are fewer SNPs to be potentially removed in the second step.

The determination of what is the best combination of the LASSO parameter $\lambda$ and the type of subsequent

Table 1 Common SNPs in model

\begin{tabular}{lccc}
\hline SNPs with MAF $>\mathbf{0 . 0 1}$ in the simulating model for Q1. Gene & SNP & MAF & SNP effect size, $\boldsymbol{\beta}$ \\
\hline$A R N T$ & C1S6533 & 0.011478 & 0.56190 \\
$F L T 1$ & $C 135431$ & 0.017217 & 0.74136 \\
$F L T 1$ & $C 135522$ & 0.027977 & 0.61830 \\
$F L T 1$ & $C 13 S 523$ & 0.066714 & 0.64997 \\
$H I F 1 A$ & $C 1451734$ & 0.012195 & 0.21203 \\
$K D R$ & $C 4 S 1878$ & 0.164993 & 0.13573 \\
$K D R$ & $C 4 S 1884$ & 0.020803 & 0.29558 \\
\hline
\end{tabular}


Table 2 Mean number of times SNPs in simulating model were selected over 200 replicates

\begin{tabular}{lcccccc}
\hline SNP & Lasso with $\boldsymbol{\lambda}_{\text {min }}$ & + AIC & + BIC & Lasso with $\boldsymbol{\lambda}_{\text {1se }}$ & + AIC & + BIC \\
\hline C1S6533 & 0.445 & 0.440 & 0.365 & 0.040 & 0.040 & 0.040 \\
C13S431 & 0.775 & 0.665 & 0.555 & 0.185 & 0.185 & 0.180 \\
C13S522 & 0.985 & 0.970 & 0.880 & 0.850 & 0.850 & 0.850 \\
C13S523 & 1.000 & 1.000 & 1.000 & 1.000 & 1.000 & 1.000 \\
C14S1734 & 0.085 & 0.065 & 0.035 & 0.000 & 0.000 & 0.000 \\
C4S1878 & 0.375 & 0.265 & 0.200 & 0.045 & 0.045 & 0.045 \\
C4S1884 & 0.425 & 0.385 & 0.285 & 0.055 & 0.055 & 0.055 \\
Total correct & 4.09 & 3.79 & 3.32 & 2.175 & 2.175 & 2.170 \\
Total selected & 28.05 & 21.18 & 14.30 & 3.29 & 3.22 & 3.10 \\
\hline
\end{tabular}

The mean number of total SNPs selected for each of the variations of the model selection procedure are also shown.

stepwise selection (AIC or BIC) depends on the context. Use of $\lambda_{1 \mathrm{se}}$ and the BIC is likely to result in identification of fewer spurious associations but also less true causal associations. If it is more important to ensure that all causal associations are captured for further investigation, then it may be better to use $\lambda_{\min }$ and the AIC.

After averaging over all 200 replicates, we found that after the LASSO step using $\lambda_{\text {min }}$, six of the seven SNPs in the simulating model were selected more often than any other SNPs that were not in the simulating model. The seventh SNP in the simulating model (C14S1734) was the 21st most selected SNP (i.e., 14 SNPs not in the simulating model were selected more often). After applying stepwise selection with the BIC, C14S1734 was the 20th most selected SNP, and the other six SNPs in the simulating model were still the most selected. Note that there is a relationship between the mean number of times that SNPs in the simulating model are selected and the MAF and effect size of those SNPs (see Table 1). The three most often selected SNPs are those in the gene FLT1 on chromosome 13. It appears to be no coincidence that these are the SNPs with the largest effect size. The two SNPs with the smallest effect size are C14S1734

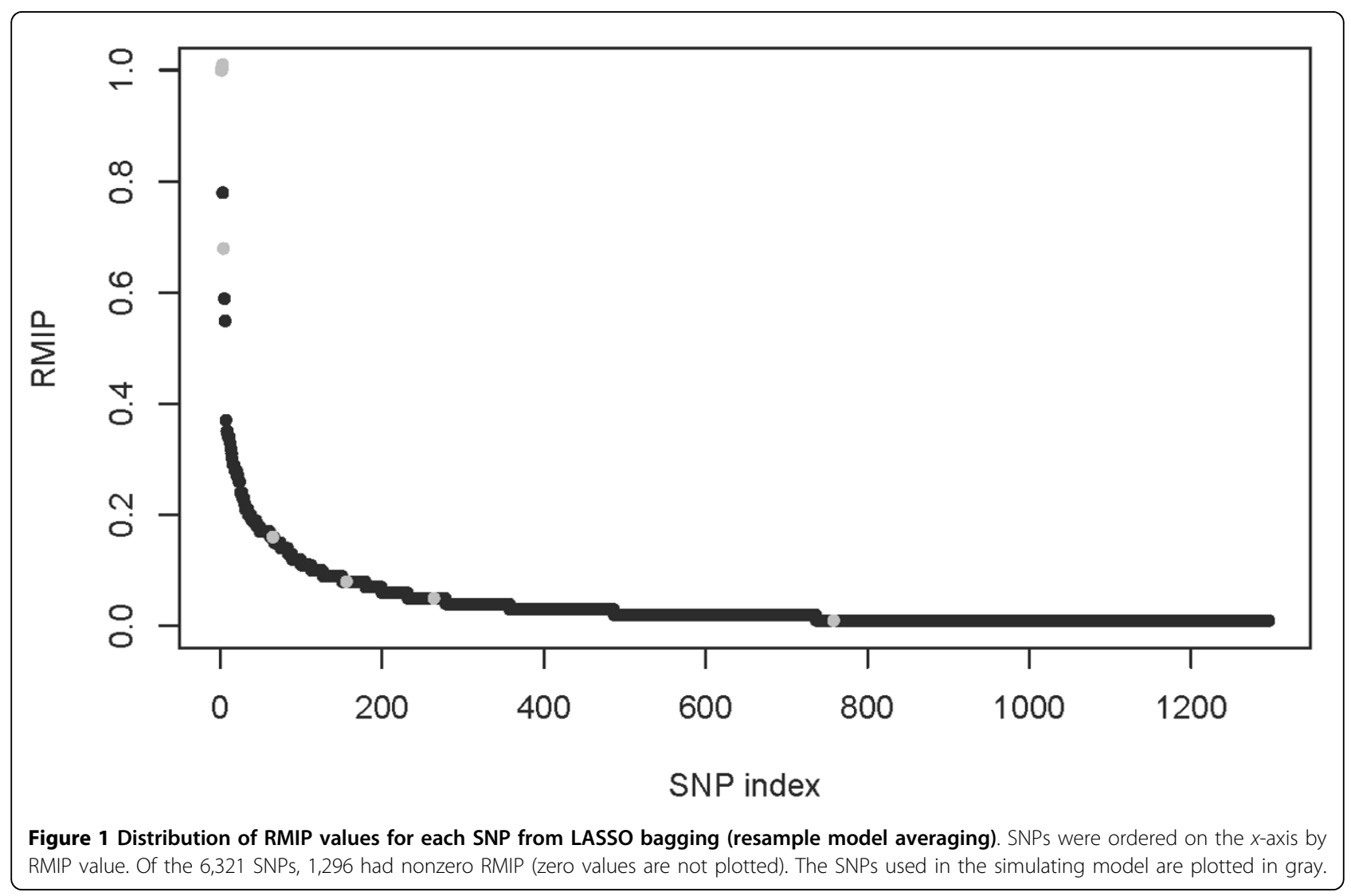


(0.21203) and C4S1878 (0.13573). Although it is C4S1878 that has the smallest effect size, it is C14S1734 that is detected by the model selection procedure least often. It appears that this is due to C14S1734 having a much smaller MAF than C4S1878 (0.012 compared to 0.165).

\section{Stability analysis}

We performed LASSO bagging with 100 resamples and fixed penalty parameter $\lambda$ over all the resamples. The distribution of the RMIP values for each SNP is plotted in Figure 1. The SNPs in the simulating model are marked. The plot shows that only three of the seven SNPs in the simulating model have RMIP $>0.5$. There are also three spurious associations detected with RMIP $>0.5$. It is important to note that here we are considering only a single replicate (replicate 1), unlike earlier results, which were obtained by averaging over all 200 replicates. This is indicative of the problems of insufficient sample size, and the resultant tradeoff between false negatives and false positives.

\section{Discussion and conclusions}

Simultaneous analysis of SNPs with penalized-regression approaches, such as the LASSO method, have gained attention recently. It is an open question as to what is the best sequence of steps when using these approaches. Here, we used the LASSO to identify a small set of SNPs that are then used as candidates in a standard model selection procedure. Recently, Cho et al. [3] proposed a procedure in which SNPs are initially filtered out based on single-SNP association before performing the penalized regression. Our investigations suggest that filtering on the basis of single-SNP association as a first step may leave out SNPs that would be selected by penalized regression.

We also note that model selection with the LASSO (using the glmnet package in $\mathrm{R}$ ) is remarkably fast. The analysis of the GAW17 data for a single phenotype replicate can be carried out in a matter of seconds on a desktop computer. The glmnet package computation time scales linearly with the number of observations, the number of covariates, and the number of selected covariates, so it is quite feasible to analyze much larger data sets. We also experimented with the hyper-LASSO procedure introduced by Hoggart et al. [1]. This procedure is promising in terms of its model selection properties, but for large data sets the computation time is substantially greater than for the LASSO.

\section{Acknowledgments}

We acknowledge funding of this research through Australian National Health and Medical Research Council grant 525453. We also thank the two anonymous reviewers. The Genetic Analysis Workshop is supported by National Institutes of Health grant R01 GM031575.
This article has been published as part of BMC Proceedings Volume 5 Supplement 9, 2011: Genetic Analysis Workshop 17. The full contents of the supplement are available online at http://www.biomedcentral.com/1753$6561 / 5$ ? issue $=59$

\section{Author details}

'Prince of Wales Clinical School, University of New South Wales, New South Wales 2052, Australia. ${ }^{2}$ School of Mathematics and Statistics, University of New South Wales, New South Wales 2052, Australia.

\section{Authors' contributions}

AJM, SG and SRW conceived the statistical analysis. AJM carried out the statistical analysis with assistance from CM. AJM drafted the manuscript. All authors read and approved the final manuscript.

\section{Competing interests}

The authors declare that they have no competing interests.

Published: 29 November 2011

\section{References}

1. Hoggart CJ, Whittaker JC, De lorio M, Balding DJ: Simultaneous analysis of all SNPs in genome-wide and re-sequencing association studies. PLOS Genet 2008, 4:e1000130.

2. Yang J, Benyamin B, McEvoy BP, Gordon S, Henders AK, Nyholt DR, Madden PA, Heath AC, Martin NG, Montgomery GW, et al: Common SNPs explain a large proportion of the heritability for human height. Nat Genet 2010, 42:565-569.

3. Cho S, Kim K, Kim YJ, Lee JK, Cho YS, Lee JY, Han BG, Kim H, Ott J, Park T: Joint identification of multiple genetic variants via elastic-net variable selection in a genome-wide association analysis. Ann Hum Genet 2010, 74:416-428.

4. Wu J, Devlin B, Ringquist S, Trucco M, Roeder K: Screen and clean: a tool for identifying interactions in genome-wide association studies. Genet Epidemiol 2010, 34:275-285.

5. Fan J, Lv J: A selective overview of variable selection in high dimensional feature space. Stat Sinica 2010, 20:101-148.

6. Tibshirani R: Regression shrinkage and selection via the lasso. J Roy Stat Soc Ser B 1996, 58:267-288.

7. Dasgupta A, Sun YV, König IR, Bailey-Wilson JE, Malley JD: A brief review of machine learning methods in genetic epidemiology: the GAW17 experience. Genet Epidemio/ 2011, X(suppl X):X-X.

8. Friedman J, Hastie T, Tibshirani R: Regularization paths for generalized linear models via coordinate descent. J Stat Softw 2010, 33:1-22.

9. Meinshausen N, Buehlmann P: Stability selection. J Roy Stat Soc Ser B 2010, 72:417-473.

doi:10.1186/1753-6561-5-S9-S24

Cite this article as: Motyer et al: LASSO model selection with postprocessing for a genome-wide association study data set. BMC Proceedings 2011 5(Suppl 9):S24.

\section{Submit your next manuscript to BioMed Central} and take full advantage of:

\footnotetext{
- Convenient online submission

- Thorough peer review

- No space constraints or color figure charges

- Immediate publication on acceptance

- Inclusion in PubMed, CAS, Scopus and Google Scholar

- Research which is freely available for redistribution
} 